\title{
FIRST POLARIMETRIC MEASUREMENTS AND MODELING OF THE PASCHEN-BACK EFFECT IN CaH TRANSITIONS
}

\author{
S. V. Berdyugina ${ }^{1}$ AND D. M. Fluri \\ Institute of Astronomy, ETH Zurich, 8092, Zurich, Switzerland; sveta@astro.phys.ethz.ch, fluri@astro.phys.ethz.ch \\ R. RAMELli AND M. BIANDA ${ }^{2}$ \\ Istituto Ricerche Solari Locarno, Via Patocchi, CH-6605 Locarno-Monti, Switzerland; ramelli@irsol.ch, mbianda@irsol.ch \\ AND \\ D. Gisler And J. O. Stenflo ${ }^{3}$ \\ Institute of Astronomy, ETH Zurich, 8092, Zurich, Switzerland; gisler@astro.phys.ethz.ch, stenflo@astro.phys.ethz.ch \\ Received 2006 March 26; accepted 2006 August 3; published 2006 September 5
}

\begin{abstract}
We report the first spectropolarimetric observations and modeling of $\mathrm{CaH}$ transitions in sunspots. We have detected strong polarization signals in many $\mathrm{CaH}$ lines from the $A-X$ system, and we provide the first successful fit to the observed Stokes profiles using the previously developed theory of the Paschen-Back effect in arbitrary electronic states of diatomic molecules and polarized radiative transfer in molecular lines in stellar atmospheres. We analyze the $\mathrm{CaH}$ Stokes profiles together with quasi-simultaneous observations in TiO bands and conclude that $\mathrm{CaH}$ provides a valuable diagnostic of magnetic fields in sunspots, starspots, cool stars, and brown dwarfs.

Subject headings: magnetic fields - molecular processes — polarization — sunspots

Online material: color figures
\end{abstract}

\section{INTRODUCTION}

$\mathrm{CaH}$ is one of the most important astrophysical molecules. Two red-band systems of $\mathrm{CaH}, A^{2} \Pi-X^{2} \Sigma^{+}$and $B^{2} \Sigma^{+}-X^{2} \Sigma^{+}$, were first detected in spectra of M dwarfs by Fowler (1907) and almost immediately after that identified in the sunspot spectrum by Olmsted (1908). Later it was established that the formation of $\mathrm{CaH}$ in stellar atmospheres is strongly influenced by gas pressure; thus, $\mathrm{CaH}$ bands, as well as other metal hydride bands, can be used as a luminosity indicator of cool stars (Öhman 1934; Mould 1976; Mould \& Wallis 1977 ; Barbuy et al. 1993). Absorption in $\mathrm{CaH}$ bands is also an important opacity source in brown dwarfs and can be used for studying these substellar objects (e.g., Burrows et al. 2001). The astrophysical value of $\mathrm{CaH}$ has motivated a series of laboratory spectroscopic studies of the molecule (e.g., Berg \& Klynning 1974a, 1974b; Martin 1984; Weck et al. 2003; Shayesteh et al. 2004; Steimle et al. 2004). Furthermore, because of its remarkable magnetic properties, $\mathrm{CaH}$ was the first molecule having been cooled down to nanokelvin temperatures to produce molecular Bose-Einstein condensation in a magnetic trap (Weinstein et al. 1998).

A laboratory study of the red $\mathrm{CaH}$ bands in a magnetic field started immediately after Hill (1929) had formulated the first theory of the Zeeman and Paschen-Back effects in molecular doublet states for the case intermediate between Hund's cases (a) and $(b)$. This theory explained well Zeeman shifts of transitions in the $A-X$ system with smaller rotational numbers. Transitions between higher rotational levels, as well as those in the $B-X$ system, were found to be affected by the rotational perturbation between the excited states $A^{2} \Pi$ and $B^{2} \Sigma^{+}$(Watson \& Bender 1930; Watson 1932; Cunningham \& Watson 1933). Despite the progress in explaining Zeeman shifts of $\mathrm{CaH}$ transitions, no spectropolarimetric measurements were made to date

\footnotetext{
${ }^{1}$ Astronomy Division, University of Oulu, P.O. Box 3000, FIN-90014 Oulu, Finland.

${ }^{2}$ Institute of Astronomy, ETH Zurich, 8092, Zurich, Switzerland.

${ }^{3}$ Faculty of Mathematics and Science, University of Zurich, Zurich, Switzerland.
}

neither in laboratories, nor in astrophysics. The only report of possible Zeeman broadening of $\mathrm{CaH}$ lines in sunspots was given by Mallia (1970).

A study of solar and stellar magnetic fields with molecular spectropolarimetry is a new and rapidly developing field pioneered by Berdyugina et al. (2000). Molecules are preferably formed in cooler environments and thus bear the information on their physical conditions, including the magnetic field. An overview of magnetic properties of astrophysically important diatomic molecules (Berdyugina \& Solanki 2002; Berdyugina et al. 2003, 2005; Asensio Ramos \& Trujillo Bueno 2006) has indicated a great potential of molecular spectropolarimetry for studying cosmic magnetic fields. $\mathrm{CaH}$ lines are especially useful diagnostic of sunspots with the umbral temperature of 4000$4250 \mathrm{~K}$, at which absorption in $\mathrm{CaH}$ is significantly stronger than that in $\mathrm{TiO}$ (Berdyugina et al. 2003). Moreover, because of this property, $\mathrm{CaH}$ lines may be more readily detected in starspots on active $\mathrm{G}-\mathrm{K}$ stars than TiO lines. A simultaneous analysis of $\mathrm{CaH}$ and $\mathrm{TiO}$ lines can therefore provide valuable constraints for temperature, pressure, and magnetic field in the atmosphere of sunspots, starspots, and cool stars.

Here we report the first spectropolarimetric measurements and modeling of $\mathrm{CaH}$ Stokes profiles. We detect strong polarization features in many $\mathrm{CaH}$ lines from the $A-X$ system observed in sunspots and successfully fit their Stokes profiles using the previously developed theory of the Paschen-Back effect (PBE) in arbitrary electronic states of diatomic molecules (Berdyugina et al. 2005) and polarized radiative transfer of molecular lines in the stellar atmospheres (Berdyugina et al. 2003). We therefore confirm that $\mathrm{CaH}$ is a valuable diagnostic of magnetic fields in cool astrophysical sources. We describe our polarimetric observations in $\S 2$. Then, in $\S 3$, we discuss the PBE in the $\mathrm{CaH}$ $A-X$ system and present calculations of the Zeeman patterns. Modeling Stokes profiles of the $\mathrm{CaH}$ lines and a comparison with observations are presented in $\S 4$. Finally, we summarize our results and draw the conclusions in $\S 5$. 

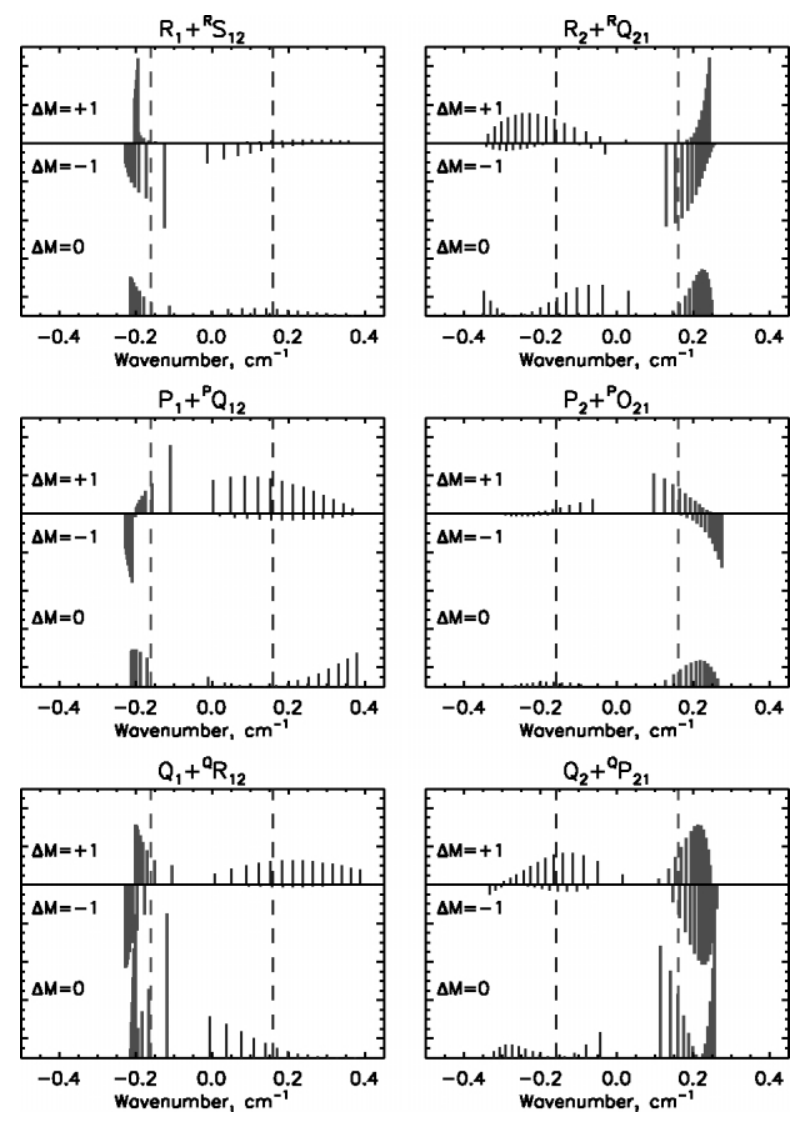

FIG. 1.-CaH magnetic patterns for different rotational branches arising from the lower rotational levels with $N=7$ at the magnetic field strength of $3 \mathrm{kG}$. The Zeeman components shown by thick and thin sticks correspond to the main and satellite lines, respectively. The components with $\Delta M=-1$ are plotted downward for clarity. Vertical dashed lines indicate the positions of the main and satellite lines in zero magnetic field with respect to the doublet center, which is at $0.0 \mathrm{~cm}^{-1}$. [See the electronic edition of the Journal for a color version of this figure.]

\section{OBSERVATIONS}

The spectropolarimetric observations were carried out at the Gregory Coudé Telescope of the Istituto Ricerche Solari Locarno (IRSOL) with the Zurich IMaging POLarimeter (ZIMPOL; Povel 2001). Due to high modulation frequency of $42 \mathrm{kHz}$ (which is much higher than typical seeing frequencies), ZIMPOL allows us to obtain measurements that are unaffected by seeing induced spurious effects. Calibration included measurements of the polarimetric efficiency, dark current, flat field, and instrumental polarization (Ramelli et al. 2005).

We observed a large spot in the active region NOAA AR 822, which passed across the solar disk on 2005 November 17-24. The slit was placed across the umbra's darkest part, and the spectra were recorded from the umbra, both sides of the penumbra, and the photosphere. The ZIMPOL system allowed simultaneous measurements of Stokes $I Q V$ or $I U V$. Both sequences were recorded for each wavelength region. Calibrations were performed as explained by Gandorfer et al. (2004). Measurements of the instrumental polarization are used to correct the data as shown by Ramelli et al. (2005). The stray light introduced by the instrumentation is estimated to be about $5 \%$. To account for this effect, calculated spectra were diluted with $5 \%$ of the synthetic photospheric spectrum.

To increase the signal-to-noise ratio, we averaged spectra along the slit area covering the central part of the umbra. A correction for the telluric line absorption was applied using the transmission spectrum from the NSO atlas by Wallace et al. (1998).

Several spectral regions with strong $\mathrm{CaH}$ lines were observed on different dates. Here we analyze two regions centered at 693.9 and $697.2 \mathrm{~nm}$, which were observed on 2005 November 17-21. The regions include lines mainly from the $A-X(0,0)$ band, which were expected to show strong polarization signals. Since $\mathrm{CaH}$ lines are blended with numerous weak $\mathrm{TiO}$ lines from the $\gamma$ system, two other regions centered at 705.7 and $759.0 \mathrm{~nm}$ were also observed. They include the $\mathrm{TiO}(0,0)$ and $(0,1)$ band heads of the $\gamma$-system. The first band is known to show strong polarization signatures (Berdyugina et al. 2000), and the second one belonging to the same electronic system is expected to have similar magnetic sensitivity. These are the first polarimetric observations of $\mathrm{CaH}$ lines and of the $\mathrm{TiO} \gamma(0,1)$ band.

\section{PASCHEN-BACK EFFECT IN THE CaH $A-X$ SYSTEM}

Recently, we presented a general numerical approach to the molecular PBE that is valid for terms of any multiplicity and accounts for interactions of all rotational levels in an electronic state intermediate between Hund's cases $(a)$ and $(b)$ (Berdyugina et al. 2005). This allows us to develop novel diagnostic tools for studying magnetic fields in astrophysical objects. $\mathrm{CaH}$ transitions are obviously among those of the first choice.

The $\mathrm{CaH} A^{2} \Pi-X^{2} \Sigma^{+}$band system is observed in the wavelength region $660-760 \mathrm{~nm}$. The ground state $X^{2} \Sigma^{+}$is well described by a pure Hund's case $(b)$, since the projection of the electronic orbital momentum on the internuclear axis $\Lambda$ is zero. Small spin-rotation interaction results in the splitting of each rotational level onto two levels of the fine structure. The spin-rotation coupling constant of $0.0436 \mathrm{~cm}^{-1}$ (Martin 1984) indicates that significant perturbations are already seen at magnetic fields stronger than $100 \mathrm{G}$, and the complete PBE in the lowest rotational level occurs at $470 \mathrm{G}$. The excited $A^{2} \Pi$ state is intermediate between Hund's cases $(a)$ and $(b)$. The relatively large spin-orbit coupling constant of $\sim 79 \mathrm{~cm}^{-1}$ and rotational constant of $\sim 4.3 \mathrm{~cm}^{-1}$ (Martin 1984) suggest that lower rotational levels are closer to Hund's case $(a)$, while higher levels approach Hund's case $(b)$ due to gradual spin uncoupling from the internuclear axis with increasing rotation. The PBE in this electronic state becomes noticeable only at magnetic fields stronger than $20 \mathrm{kG}$ and in higher rotational levels.

Rotational levels in the $A^{2} \Pi$ vibrational states with $v \geq 1$ perturb the nearby levels of the $B^{2} \Sigma^{+}$state and lend some of their properties to the $B$ state. This perturbation also results in $\Lambda$-doubling of the $A$ state. Accounting for this perturbation should result in different magnetic properties of transitions of different parities. This is left out in the present consideration and will be investigated in the future.

Using molecular constants determined by Berg \& Klynning (1974a) and Martin (1984) and the PBE theory as described by Berdyugina et al. (2005), we calculated wavelength shifts and strengths of Zeeman components for all $\mathrm{CaH}$ lines in the observed spectral regions. Since the PBE results in the appearance of forbidden transitions ( $\mathrm{O}$ and $\mathrm{S}$ branches), they were also included in the calculation. Their zero-field wavelengths were predicted using those of allowed transitions measured in the laboratory by S. Davis (2000, private communication). Examples of Zeeman patterns for different main and satellite rotational branches are shown in Figure 1. Note that the PBE noticeably alters relative strengths of transitions with different $\Delta M$, resulting in strongly asymmetric patterns and wavelength shifts of the line center depending on the magnetic field 

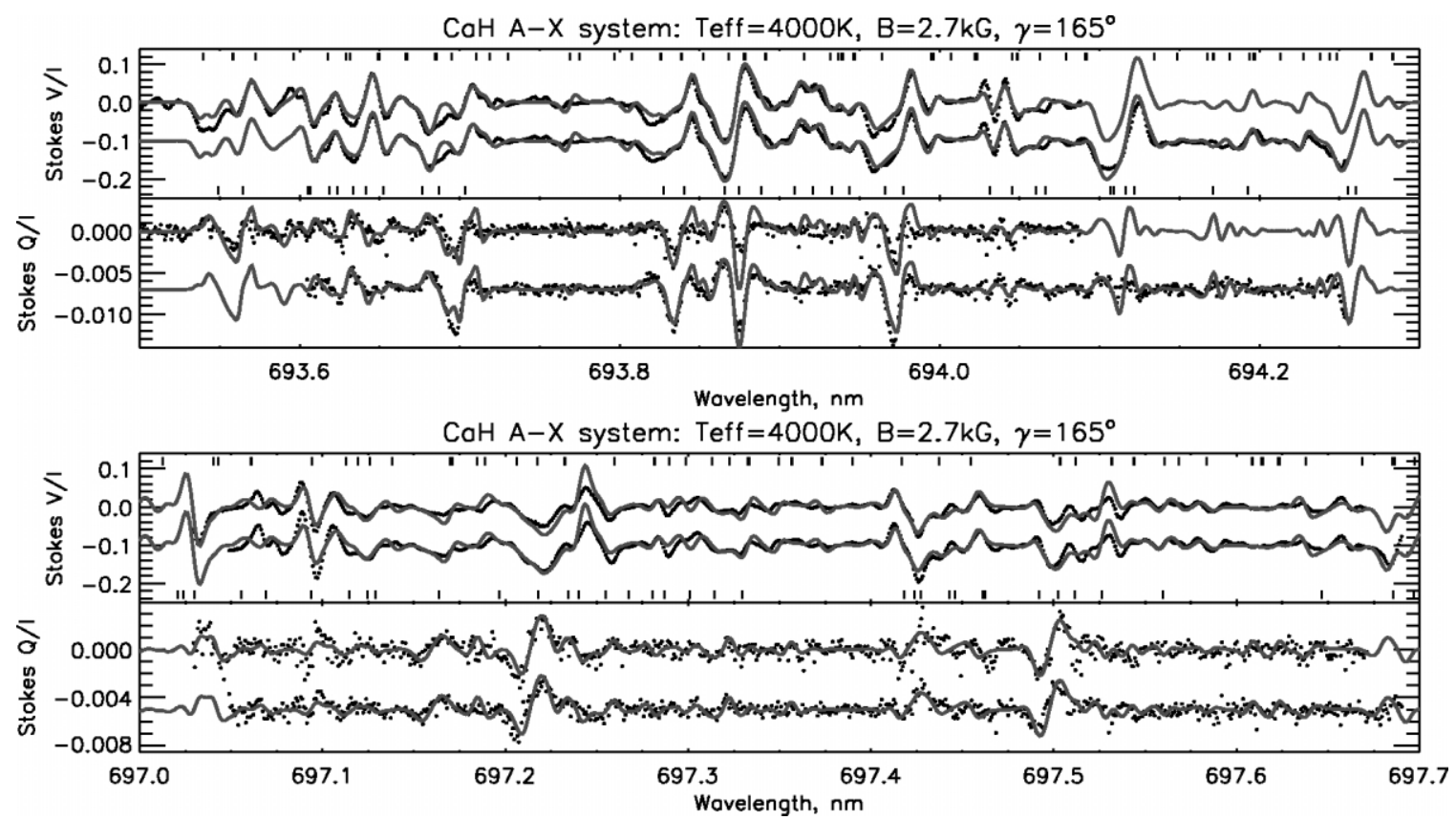

FIG. 2.- $\mathrm{CaH}$ Stokes profiles in the sunspot umbra. Dots represent observations: two different dates for each region, one being shifted in vertical direction for clarity. Solid lines are synthetic spectra. The $693.9 \mathrm{~nm}$ region was observed on 2005 November 18 and 19, the other one on 2005 November 17 and 19 . In the top panels, vertical dashes indicate positions of $\mathrm{TiO}$ lines (top edge) and $\mathrm{CaH}$ lines (bottom edge). The effective temperature of the equilibrium model and the magnetic field strength and angle to the line of sight indicated above the plots have been chosen to fit reasonably both pairs of observations. [See the electronic edition of the Journal for a color version of this figure.]

strength. Also, the total strength of the main branch lines decreases, while that of satellite and forbidden lines increases.

\section{STOKES PROFILES}

The polarized radiative transfer in all four Stokes parameters for molecular lines in stellar atmospheres was solved according to the description given by Berdyugina et al. (2003). The Stokes parameter synthesis was done using equilibrium stellar atmosphere models by Kurucz (1993).

In the two $\mathrm{CaH}$ spectral regions (Fig. 2) the strongest lines are from the least perturbed vibrational band $(0,0)$ of the $\mathrm{CaH}$ $A-X$ system. A number of weaker lines from the $(1,1)$ and $(2$, 2) bands are also present. In addition to the $\mathrm{CaH}$ lines, many $\mathrm{TiO}$ lines from the $\gamma$-system were included in the spectrum synthesis. These lines also show a remarkable Zeeman effect (Berdyugina et al. 2000), but here they originate from higher vibrational states and appear weaker than $\mathrm{CaH}$ lines. Also, a few atomic and $\mathrm{MgH} B^{\prime}-X$ blends were included in the line list. Despite the complexity of various line contributions, there is a remarkable resemblance between the observed and calculated
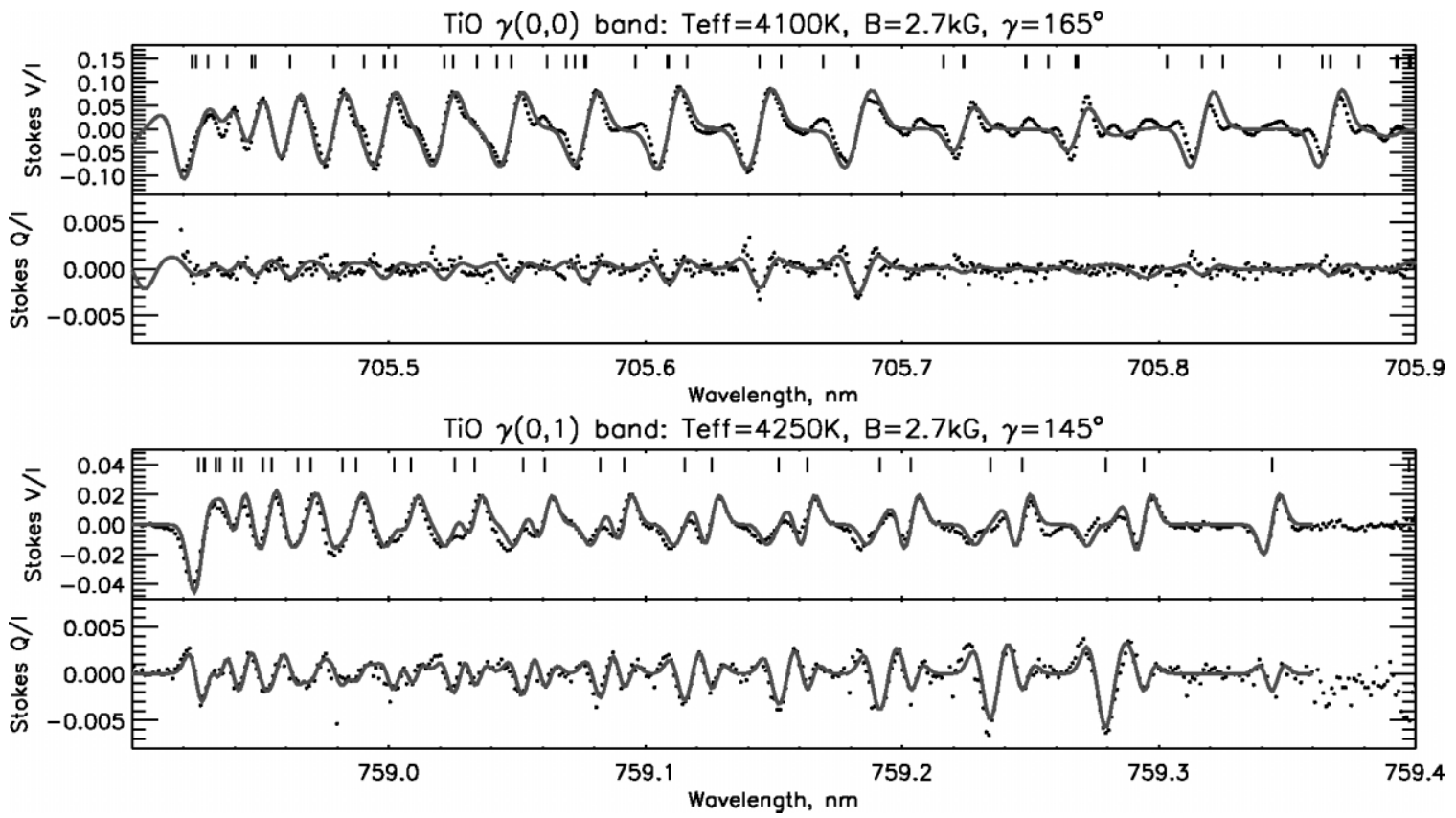

FIG. 3.-Same as Fig. 2, but for TiO Stokes profiles. The $705.7 \mathrm{~nm}$ region was observed on 2005 November 19, and the $759.0 \mathrm{~nm}$ on $2005 \mathrm{November} 21$. [See the electronic edition of the Journal for a color version of this figure.] 
Stokes profiles (Fig. 2). This implies that our PBE calculation for the $\mathrm{CaH} A-X$ system is adequate for transitions with minimal perturbation by the $B$ state. Small discrepancies in the fits are mainly due to missing blends but can also be due to the neglected perturbation. The peculiar Stokes profiles caused by the PBE are extremely sensitive to the magnetic field strength.

The regions with the $\mathrm{TiO}$ bands are only slightly blended by other lines. At sunspot magnetic fields the TiO transitions are well described by the Zeeman regime calculations for the intermediate Hund's case $(a-b)$ (Berdyugina \& Solanki 2002; Berdyugina et al. 2003). Prominent polarization signatures are observed in both bands (Fig. 3): Stokes $V$ is the strongest for the near disk center observation (705.7 $\mathrm{nm}$ region), while Stokes $Q$ becomes comparable to Stokes $V$ for the off center observation (759.0 $\mathrm{nm}$ region).

The high sensitivity to the temperature and magnetic field strength of the $\mathrm{CaH}$ and $\mathrm{TiO}$ Stokes profiles allowed us to constrain these parameters in the umbra. Atmosphere models with somewhat different effective temperatures were employed to obtain good fits to the $\mathrm{CaH}$ and $\mathrm{TiO}$ profiles in different spectral regions. This indicates that simple equilibrium models are not able to reproduce the complex structure of sunspot umbrae revealed by molecular lines, and a more sophisticated, multicomponent analysis based on inversion techniques is needed. Our analysis suggests that simultaneous spectropolarimetry in $\mathrm{CaH}$ and $\mathrm{TiO}$ lines with high spatial resolution would be essential for revealing the internal structure of the sunspot umbra and for understanding its heating mechanisms.

\section{CONCLUSIONS}

First spectropolarimetric observations of the $\mathrm{CaH} A^{2} \Pi-$ $X^{2} \Sigma^{+}$system have revealed strong polarization signatures in many lines. Peculiar Stokes profiles observed in sunspots are caused mainly by the Paschen-Back effect on the fine structure of the ground state which significantly perturbs the internal molecular structure at fields stronger than $\sim 100 \mathrm{G}$.

Our advanced calculation of the molecular PBE combined with the full Stokes radiative transfer provided the first successful fit to the observed $\mathrm{CaH}$ Stokes profiles. This opens a new opportunity for studying magnetic fields in sunspots and starspots and also in very cool stars and brown dwarfs.

We are thankful to Sumner Davis for providing laboratory wavelengths of $\mathrm{CaH}$ transitions. The observations at IRSOL are possible thanks to the financial support that has been provided by the canton of Ticino, the city of Locarno, ETH Zurich, and the Fondazione Carlo e Albina Cavargna. S. V. B. acknowledges the EURYI award from the ESF, SNF grants PE002-104552 and 200021-103696, and ETH research grant TH-2/04-3.

\section{REFERENCES}

Asensio Ramos, A., \& Trujillo Bueno, J. 2006, ApJ, 636, 548

Barbuy, B., Schiavon, R. P., Gregorio-Hetem, J., Singh, P. D., \& Batalha, C. 1993, A\&AS, 101, 409

Berdyugina, S. V., Braun, P. A., Fluri, D. M., \& Solanki, S. K. 2005, A\&A, 444, 947

Berdyugina, S. V., Frutiger, C., Solanki, S. K., \& Livingston, W. 2000, A\&A, 364, L101

Berdyugina, S. V., \& Solanki, S. K. 2002, A\&A, 385, 701

Berdyugina, S. V., Solanki, S. K., \& Frutiger, C. 2003, A\&A, 412, 513

Berg, L.-E., \& Klynning, L. 1974a, Phys. Scr. 10, 331 1974b, A\&AS, 13, 325

Burrows, A., Hubbard, W. B., Lunine, J. I., \& Liebert, J. 2001, Rev. Mod. Phys., 73, 719

Cunningham, W. P., \& Watson, W. W. 1933, Phys. Rev., 44, 815

Fowler, A. 1907, Proc. R. Soc. London A, 79, 509

Gandorfer, A. M., et al. 2004, A\&A, 422, 703

Hill, E. L. 1929, Phys. Rev., 34, 1507

Kurucz, R. 1993, CD-ROM 13, ATLAS9 Stellar Atmosphere Programs and $2 \mathrm{~km} / \mathrm{s}$ Grid (Cambridge: SAO)

Mallia, E. A. 1970, Sol. Phys., 14, 125

Martin, H. 1984, J. Mol. Spectrosc., 108, 66

Mould, J. R. 1976, ApJ, 207, 535
Mould, J. R., \& Wallis, R. E. 1977, MNRAS, 181, 625

Öhman, Y. 1934, ApJ, 80, 171

Olmsted, C. M. 1908, ApJ, 27, 66

Povel, H. P. 2001, in ASP Conf. Ser. 248, Magnetic Fields across the HerzsprungRussell Diagram, ed. G. Mathys, S. K. Solanki, \& D. T. Wickramasinghe (San Francisco: ASP), 543

Ramelli, R., Bianda, M., Trujillo Bueno, J., Merenda, L., \& Stenflo, J. O. 2005, in Chromospheric and Coronal Magnetic Fields, ed. D. E. Innes, A. Lagg, \& S. K. Solanki (ESA SP-596; Noordwijk: ESA), 82

Shayesteh, A., Walker, K. A., Gordon, I., Appadoo, D. R. T., \& Bernath, P. F. 2004, J. Mol. Struct., 695, 23

Steimle, T. C., Gengler, J., \& Chen, J. 2004, Canadian J. Chem., 82, 779

Wallace, L., Livingston, W. C., Bernath, P. F., \& Ram, R. S. 1998, An Atlas of the Sunspot Umbral Spectrum in the Red and Infrared from 8900 to 15 $050 \mathrm{~cm}^{-1}$ (6642 to $11230 \AA$ ) (Tucson: NOAO), ftp://ftp.noao.edu/fts/ spot3atl

Watson, W. W. 1932, Phys. Rev., 39, 278

Watson, W. W., \& Bender, W. 1930, Phys. Rev., 35, 1513

Weck, P. F., Stancil, P. C., \& Kirby, K. 2003, J. Chem. Phys., 118, 9997

Weinstein, J. D., deCarvalho, R., Guillet, T., Friedrich, B., \& Doyle, J. M. 1998, Nature, 395, 148 